\title{
Singularities of Bethe Ansatz via Robinson Numbers
}

\author{
A. Koper ${ }^{a, *}$, P. KRAsoñ ${ }^{b}$ AND J. Milewski ${ }^{c}$ \\ ${ }^{a}$ Department of Physics, Adam Mickiewicz University, Poznań 61-614, Poland \\ ${ }^{b}$ Department of Mathematics and Physics, Szczecin University, Wielkopolska 15, 70-415 Szczecin, Poland \\ ${ }^{c}$ Institute of Mathematics Poznań University of Technology, Piotrowo 3A, 60-965 Poznań, Poland \\ In this work we suggest a rigorous mathematical approach for explanation of singular solutions of Bethe Ansatz \\ by means of Robinson complex hypernumbers. There are several approaches towards these singular solutions eg. \\ formal infinitesimals or germs of meromorphic functions. Our aim is to make them precise using non-standard \\ analysis and show that they are essentially equivalent.
}

DOI: 10.12693/APhysPolA.133.438

PACS/topics: 75.10.Jm, 02.40.Xx, 02.10.De, 85.75.Ff

\section{Introduction}

Hyperreal numbers since their development [1] found numerous physical applications [2]. There are also applications in probability in analysis of random walks, Brownian motions cf. [3] and Feynman path integrals [4]. In the present paper we apply non-standard analysis to the system of algebraic equations connected to Bethe Ansatz. We obtain a family of solutions to singular Bethe equations by means of arithmetic of hyperreal numbers, which is the novelty of the paper. The solutions to these equations were found independently in [5] by means of germs of meromorphic functions and in [6] by formal infinitesimals. Our new non-standard solution here has necessary mathematical precision and explain the nature of the singularities $[5,6]$.

\section{Remarks on non-standard analysis}

Non-standard analysis was developed by A. Robinson cf. [1]. It answers the need of mathematicians and physists for recognising different infinitesimal as well as "arbitrarily large" quantities. Such a need was already present in Leibniz philosophy of mathematics and mathematical analysis in particular cf. [7]. These ideas were also present in the works of other mathematicians e.g. L. Euler, T. Skolem and S. Łoś. T. Skolem discovered existence of the non-standard arithmetic. However, it was A. Robinson who constructed an extension (enlargement) of natural, real and complex numbers and then developed the usual analytical tools that are now known as non-standard analysis. The original construction uses the advanced mathematical logic and set theory, especially the theory of higher order languages and the theory of ultrapowers cf. [1, 7]. The great achievement of A. Robinson was showing that the new theory is consistent and non-contradictory. One can think [3] of hyperreals as the model that comes by adding a predicate "st" for standard (usual numbers) and three new axioms to the

*corresponding author; e-mail: andkoper@amu.edu.pl theory. These axioms along with all standard properties of the standard part of the theory govern the nonstandard analysis. From physical point of view these information is sufficient and one is more interested in basic properties and rules of operation on Robinson hyperreal numbers $\mathbb{R}_{h}$. We briefly state them here. For an excellent expositions on this see [3] or [1]. An infinitely small or infinitesimal real number $\epsilon$ is a number such that its absolute value $|\epsilon|$ is smaller than any number from the sequence $\frac{1}{n}$, where $n$ is a standard positive integer. Similarly, a number $\eta$ is infinitely large if it is larger than any standard natural number. A number $r \in \mathbb{R}_{h}$ is called $f$ nite if $|r|<m$ for some standard natural number $m$. For better understanding of the non-standard part define the halo of zero by the formula

$$
\operatorname{hal}(0)=\left\{x \in \mathbb{R}_{h}: x \text { is infinitely small }\right\} .
$$

Notice that hal $(0)$ has the following properties:

a) the only standard number in $\operatorname{hal}(0)$ is 0 ,

b) hal $(0)$ is bounded with no least upper bound.

In agreement with intuition one can view infinitely large hyperreals as inverses of nonzero elements of hal $(0)$.

In addition the field $\mathbb{R}_{h}$ is totally ordered, but contrary to the standard field of reals is non-Archimedean. Thus we see that not all properties of standard real numbers carry over to the hyperreals.

Every finite real number $x$ can be written as

$$
x=\operatorname{st}(x)+\epsilon_{x},
$$

where $\operatorname{st}(x)$ is the standard part of $x$ and $\epsilon_{x} \in \operatorname{hal}(0)$ is the non-standard one.

If $x=\operatorname{st}(x)+\epsilon_{x}$ and $y=\operatorname{st}(y)+\epsilon_{y}$ are two finite hyperreals then the operations of addition and multiplication are as follows

$$
\begin{aligned}
& x+y=(\operatorname{st}(x)+\operatorname{st}(y))+\left(\epsilon_{x}+\epsilon_{y}\right) \\
& x \cdot y=(\operatorname{st}(x) \cdot \operatorname{st}(y)) \\
& \quad+\left(\operatorname{st}(x) \cdot \epsilon_{y}+\operatorname{st}(y) \cdot \epsilon_{x}+\epsilon_{x} \cdot \epsilon_{y}\right) .
\end{aligned}
$$$$
\text { and }
$$

One also introduces an equivalence relation among elements of $\mathbb{R}_{h}$ 
$x \cong y$ iff $x-y \in \operatorname{hal}(0)$.

This relation is crucial to define the basic notions of nonstandard analysis such as convergence, derivative, integral etc, Let us remark at the end of this section that from formulae (3) an (4) it readily follows that the set of finite hyperreals $\mathbb{R}^{f} \subset \mathbb{R}_{h}$ is a ring, hal(0) an ideal of $\mathbb{R}^{f}$ and one has an isomorphism

$$
\mathbb{R}^{f} / \operatorname{hal}(0) \rightarrow \mathbb{R}, \quad x \rightarrow \operatorname{st}(x)
$$

\section{Singularities of Bethe Ansatz}

For a homogenous isotropic Heisenberg ring the system of Bethe equations [8] has the following form

$$
a_{\alpha}^{N}=(-1)^{r-1} \prod_{\beta \in \widetilde{r} \backslash\{\alpha\}} \frac{a_{\alpha} a_{\beta}-2 a_{\alpha}+1}{a_{\alpha} a_{\beta}-2 a_{\beta}+1}, \quad \alpha \in \widetilde{r},
$$

where $\widetilde{r}=\{1, \ldots, r\}$. Notice that the product is single, over $\beta$. $N$ denotes the number of nodes and $r$ the number of deviations.

In particular for the two deviation case $(r=2)$ the parameters $a, b$ satisfy the Bethe system of equations:

$$
\alpha^{N}=-\frac{a b-2 a+1}{a b-2 b+1} \quad, \quad b^{N}=-\frac{a b-2 b+1}{a b-2 a+1} .
$$

The wave function is expressed by them in the following way:

$$
\begin{aligned}
& \Psi\left(j_{1}, j_{2}\right)=(a b-2 a+1) a^{j_{1}} b^{j_{2}} \\
& -(a b-2 b+1) a^{j_{2}} b^{j_{1}},
\end{aligned}
$$

where $j_{1}, j_{2}$ denote position of deviations in the YangBaxter map:

$$
1 \leqslant j_{1}<j_{2} \leqslant N
$$

For explanation of the singularities of Bethe parameters of the bound states on the boundary of the Brillouin zone (B) it is convenient to consider the Inverse Bethe Ansatz (IBA). For this we look at the equations for pseudomomenta and energy.

$$
\begin{aligned}
& a b=\omega^{-k}, \omega=e^{2 \pi \mathrm{i} / N} \\
& a+a^{-1}+b+b^{-1}=E_{k, m} .
\end{aligned}
$$

This gives the following quadratic equation for the Bethe parameters

$$
\left(1+\omega^{k}\right) x^{2}-E x+\left(1+\omega^{-k}\right)=0 .
$$

The solutions for $x$ with given $k, E$ yield the Bethe portions of phase (the energy level is here shifted by 4 ). The method of obtaining these parameters is the IBA for the two-magnon case $[9,10]$.

The energy of the bound state for $k=0$ is $E=2$. IBA gives the following equations

$$
a b=-1, \quad a+1 / a+b+1 / b=2 .
$$

This system is contradictory (in $\mathbb{C}$ ) and therefore has no complex solution. In fact, if we pass to complex hypernumbers and view (14) as the following modifications

$$
\operatorname{st}(a b)=-1, \quad \text { st }(a+1 / a+b+1 / b)=2 .
$$

we can obtain a precise solution. The solution is given by assuming

$$
a=t+u, \quad b=-1 / t+2,
$$

where $t \in \operatorname{hal}_{\mathbb{C}}(0), u=o(t)$. Here $\operatorname{hal}_{\mathbb{C}}(0)$ denotes the complexification of hal(0). Observe that in the paper [5] this solution was given by means of germs of meromorphic functions.

The base of the Fourier wavelets for a sector of two spin deviations is a family of vectors:

$$
\left.\left|F, r, k, \boldsymbol{t}_{\alpha}\right| \Rightarrow \sum_{j \in \widetilde{N_{t}}} \omega^{-k j}\left|Q, r, \boldsymbol{t}_{\alpha}, j\right|,\right\rangle k \in B .
$$

This family is indexed by the wave vectors $k \in B$, and relative configurations $\boldsymbol{t}_{\alpha}=(\alpha, N-\alpha), 1 \leqslant \alpha \leqslant\left[\frac{N}{2}\right]$, where for an odd $k \in B$ the integer $\alpha \neq \frac{N}{2}$. Further $N_{t}=N$ for $\alpha \neq N / 2$ and $N_{t}=N / 2$ in the contrary.

It is worth observing that on a boundary of the Brillouin zone, $k=\frac{N}{2}$, the value of the phase factor in (17) is $\omega^{-k}=-1$.

For the two spin deviations on the boundary of the Brillouin zone, the Hamiltonian in the base of wavelets has the following diagonal form

$$
\hat{H}_{N, 2}^{M}=\operatorname{diag}[2,0, \ldots, 0] \text {. }
$$

$\left(M-1-r_{k}\right)$-times degenerated null eigenvalues correspond to the scattered states, whereas the value 2 corresponds to the bound state. Here $M=\frac{N}{2}$ and $r_{k}=0$ for even $k \in B$ and $r_{k}=1$ for $k$ odd. The eigenfunction for a bound state is given by means of the wavelet of the nearest neighbour:

$$
\Psi=A\left|F, r=2, k=M, \boldsymbol{t}_{1}\right\rangle,
$$

where $A$ is a suitable complex constant. Equivalently,

$$
|\Psi\rangle=A\left[\sum_{j=1}^{N-1}(-1)^{j}|j, j+1\rangle+|1, N\rangle\right] .
$$

Hence

and

$$
\Psi(j, j+1)=(-1)^{j} A, \quad \Psi(1, N)=A
$$

$$
\Psi\left(j_{1}, j_{2}\right)=0
$$

for other case. On the other hand (9) for the singular case takes the form

$$
\begin{aligned}
& \Psi\left(j_{1}, j_{2}\right)=\operatorname{st}\left[(a b-2 a+1) a^{j_{1}} b^{j_{2}}\right. \\
& \left.\quad-(a b-2 b+1) a^{j_{2}} b^{j_{1}}\right] .
\end{aligned}
$$

Comparison of the two forms of the eigenstate yields more exact approximation of the quantity $u$. In order to vanish the term

$$
\text { st }\left[(a b-2 a+1) a^{j} b^{j+1}\right]=0
$$

for $(j, j+1)$ in $(23)$ we assume that the quantity $u$ is greater than 2 with respect to $t$. Hence

$$
\Psi(j, j+1)=\operatorname{st}\left[-(a b-2 b+1) a^{j+1} b^{j}\right]
$$

and finally

$$
\Psi(j, j+1)=\operatorname{st}\left[2 b a^{j+1} b^{j}\right]=-2(-1)^{j} .
$$

Thus in (21) $A=-2$. Similarly, $\Psi(1, N)=\operatorname{st}(a b-2 a+1)$ $a b^{N}$ which gives

$$
\Psi(1, N)=\operatorname{st}\left(-\frac{u}{t^{N}}\right) .
$$

From (21) and (25) one obtains 


$$
u=K t^{N}+o\left(t^{N}\right), \quad K=2 .
$$

Nepomechie and Wang in their work [6] use formal infinitesimals. Observe that their solutions can be obtained from ours, given by means of suitable germs or hypernumbers, by means of Cayley transformation which connect spectral parameters and portions of phases:

$$
a, b=\frac{\lambda_{a, b}+\frac{\mathrm{i}}{2}}{\lambda_{a, b}-\frac{\mathrm{i}}{2}} .
$$

The inverse transformation gives the following values (cf. [6])

$$
\lambda_{1}=\lambda_{b}=\frac{\mathrm{i}}{2}+\epsilon+c \epsilon^{N}, \quad \lambda_{2}=\lambda_{a}=-\frac{\mathrm{i}}{2}+\epsilon,
$$

where $c=2 \mathrm{i}^{N+1}$. This value of $c$ follows from the fact that $K=2$ in (26).

\section{Conclusions}

The singularities of the Bethe system lead to its modification:

$$
\begin{aligned}
& \mathrm{P}(a b)=-1, \quad \mathrm{P}(a+1 / a+b+1 / b)=2 . \\
& \Psi\left(j_{1}, j_{2}\right)=\mathrm{P}\left[(a b-2 a+1) a^{j_{1}} b^{j_{2}}\right. \\
& \left.\quad-(a b-2 b+1) a^{j_{2}} b^{j_{1}}\right],
\end{aligned}
$$

where $\mathrm{P}$ denotes a suitable projection operator from the enlarged analytic-algebraic system (eg. formal infinitesimals, situable germs or complex hypernumbers). The use of non-standard analysis provides the Robinson complex numbers as the enlarged system and standarisation as the projection operator. In [5] as an extended system was choosen the family of germs of meromorphic functions defined in a neighbourhood of zero and the projection was an evaluation of a germ at zero. One does not need to analyse the nature of infinitely small quantities. In this case $P$ corresponds to passing to the zero limit for the infinitesimals.

\section{Acknowledgments}

The third author was partially sponsored by the grant 04/43 PS PB /0087 of Ministry of Science and High Education of Poland.

\section{References}

[1] A. Robinson, Non-standard Analysis (revised ed.), Princeton University Press, 1996.

[2] S. Albeverio at al., Nonstandard Methods in Stochastic Analysis and Mathematical Physics, Academic Press, 1986.

[3] F. Diener, M. Diener, Nonstandard Analysis in Practice, Springer, 1995.

[4] F. Herzberg, Stochastic Calculus with Infinitesimals, Springer, 2013.

[5] J. Milewski, Rep. on Math. Physics 70, 345 (2012).

[6] R.I. Nepomechie, Ch. Wang, J. Phys. A: Math. Theor. 46, 325002 (2013).

[7] R. Goldblatt, Lectures on the Hyperreals, An Introduction to Nonstandard Analysis, GTM 188, Springer, 1998.

[8] H. Bethe, Z. Physik 71, 205 (1931).

[9] J. Milewski, G. Banaszak, T. Lulek, M. Łabuz, Physica B 406520 (2011).

[10] G. Banaszak, B. Lulek, T. Lulek, J. Milewski, B. Szydło, Rep. Math. Phys. 71, 205 (2013). 